\title{
A Reaction-Diffusion Transport Model to Predict Precursor Uptake and Spatial Distribution in Vapor Phase Infiltration Processes
}

Yi Ren ${ }^{\mathrm{a}, \dagger}$, Emily K. McGuinness ${ }^{\mathrm{b}, \dagger}$, Chaofan Huang ${ }^{\mathrm{c}}$, V. Roshan Joseph ${ }^{\mathrm{c}}$, Ryan P. Lively ${ }^{\mathrm{a}, *}$, Mark D. $\operatorname{Losego}{ }^{\mathrm{b}, *}$

${ }^{a}$ School of Chemical and Biomolecular Engineering, Georgia Institute of Technology, Atlanta, GA 30332, United States

${ }^{\mathrm{b}}$ School of Materials Science and Engineering, Georgia Institute of Technology, Atlanta, GA 30332, United States

${ }^{\mathrm{c}}$ H. Milton Stewart School of Industrial and Systems Engineering, Georgia Institute of Technology, Atlanta, GA 30332, United States

†These authors contributed equally to the work

Corresponding Authors: ryan.lively@ chbe.gatech.edu and losego@gatech.edu

\section{Supporting Information}

\section{Table of Contents}

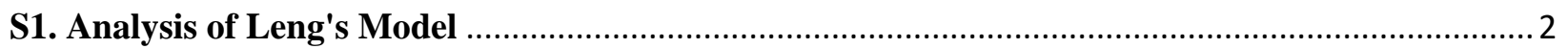

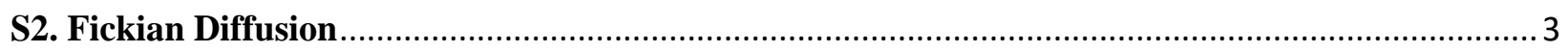

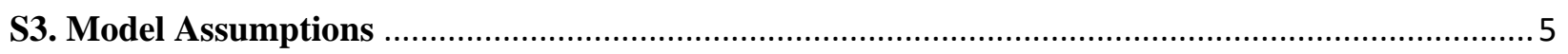

S4. Boundary Conditions for Reaction-Diffusion Model............................................................ 7

S5. Non-dimensionalization of Fickian Diffusion Curves............................................................. 8

S6. Non-dimensionalization of the Reaction-Diffusion Transport Model for VPI............................. 9

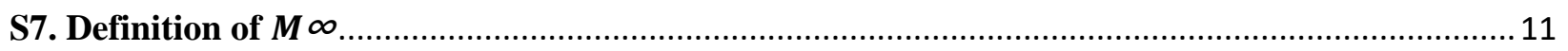

S8. Analysis of QCM Data for Experimental VPI Runs........................................................... 12

S9. Full VPI Experiments and Pressure Curves .................................................................. 13

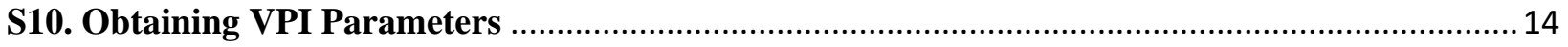




\section{S1. Analysis of Leng's Model}

Prior work into VPI model development was conducted by Leng et al. where an effective diffusivity term was used to account for instantaneous reaction:

$\frac{\partial C}{\partial t}=D \frac{\partial^{2} C}{\partial x^{2}}-\frac{\partial S}{\partial t}=\frac{D}{R+1} \frac{\partial^{2} C}{\partial x^{2}}$

where $C$ is the concentration of free diffusing species, $S$ is the concentration of the immobilized product, and due to instant reaction, an equilibrium between $S$ and $C$ is reached: $S=R \cdot C$, with $R$ being the equilibrium coefficient. ${ }^{1}$ With this model, Leng et al. were able to capture how when $R$ increases, the free diffusing $C$ is consumed faster. In this work, a larger $R$ is shown to reduce overall VPI kinetics and decrease VPI infiltration rate. However, this is not representative of all VPI systems. If only $C$, the concentration of free diffusing precursor is of interest in VPI, the statement will be correct, since higher reaction means higher consumption while diffusing, as captured by $\frac{D}{R+1}$. However, both $C$ and $S$ should be considered for the mass uptake, and since $S=$ $R \cdot C$, for the total mass uptake of $C+S$, a higher $R$ would be beneficial for the VPI kinetics and infiltration rate. Since the kinetics for diffusing of $C$ is pure Fickian with an effective diffusivity of $\frac{D}{R+1}$, the kinetics for $C+S$ will also be pure Fickian as $S$ is a scalar multiple of $C$.

The model by Leng also does not account for the fact that diffusivity is hindered by immobilized product, like all other models. There is indeed the effective diffusivity in this model, but it only accounts for the fact that $C$ is consumed and converted to $S$, but not how the formation of $S$ will decrease diffusivity. Building upon this work for the newly developed reaction-diffusion model, not only the consumption of $C$ and formation of $S$ are considered, but also the fact that diffusivity changes with hybridization of the polymer is emphasized. 


\section{S2. Fickian Diffusion}

In Fickian diffusion scenario, the governing equation for transient diffusion is shown below:

$\frac{\partial C}{\partial t}=D \frac{\partial^{2} C}{\partial x^{2}}$

where $C$ is the concentration of the metal-organic vapor, $x$ is the distance into the polymer film, and $D$ is the constant Fickian diffusion coefficient. The initial and boundary conditions are given as follows:

$C=0, @-\ell<x<\ell, t=0$

$C=C_{s}, @ x= \pm \ell, t>0$

$\frac{\partial C}{\partial x}=0, @ x=0, t>0$

This partial differential equation can be solved either analytically or numerically. The analytical solution is provided by Crank: ${ }^{2}$

For short time convergence (faster convergence with fewer terms if the diffusion length-scale is short, such as in thin films):

$\frac{C}{C_{s}}=\sum_{n=0}^{\infty}(-1)^{n} \operatorname{erfc}\left(\frac{(2 n+1) \ell-x}{2 \sqrt{D t}}\right)+\sum_{n=0}^{\infty}(-1)^{n} \operatorname{erfc}\left(\frac{(2 n+1) \ell+x}{2 \sqrt{D t}}\right)$

For long time convergence (faster convergence with fewer terms if the diffusion length-scale is long, such as in bulk polymers):

$\frac{C}{C_{s}}=1-\frac{4}{\pi} \sum_{n=0}^{\infty} \frac{(-1)^{n}}{2 n+1} \exp \left\{\frac{-D(2 n+1)^{2} \pi^{2} t}{4 \ell^{2}}\right\} \cos \left(\frac{(2 n+1) \pi x}{2 \ell}\right)$

From these equations, the concentration depth profiles over a range of times can be predicted.

Integrating the concentration over the entire polymer thickness gives the mass uptake, solving analytically by Crank ${ }^{2}$ :

For short time convergence

$\frac{M_{t}}{M_{\infty}}=2 \sqrt{\frac{D t}{\ell^{2}}}\left[\frac{1}{\sqrt{\pi}}+2 \sum_{n=1}^{\infty}(-1)^{n} i \operatorname{erfc}\left(\frac{n \ell}{\sqrt{D t}}\right)\right]$

For long time convergence

$\frac{M_{t}}{M_{\infty}}=1-\sum_{n=0}^{\infty} \frac{8}{(2 n+1)^{2} \pi^{2}} \exp \left(\frac{-D(2 n+1)^{2} \pi^{2} t}{4 \ell^{2}}\right)$ 
With the analytical solutions provided by Crank, mass uptake can be predicted at a given time for Fickian diffusion. All the above partial differential equations can also be solved numerically and would result in the same concentration and mass uptake response. 


\section{S3. Model Assumptions}

A detailed explanation of the model assumptions is provided. Firstly, an irreversible reaction is assumed. Adding a reversible reaction term would add an extra dimension of complexity to the process; it is not clear if this level of complexity is warranted. Accounting for 2 reaction rates, forward and reverse, is a nascent area of research with initial efforts being led by the Segal-Peretz group. ${ }^{3}$

Secondly, the reaction-diffusion transport model assumes no byproduct formation. If byproduct gases are formed, it will result in not only the free diffusing species but also the byproduct leaving the polymer. An extra term that accounts for by-product formation, as well as a term for by-product diffusion can be included. Due to the relative mechanistic uncertainty in VPI reactions with some processes resulting in byproducts and some not, this term has been excluded for simplicity.

Thirdly, polymer relaxation is not modeled in this section. Polymer relaxation is a common phenomenon in gas sorption of glassy polymers. In a glass polymer, Fickian diffusion occurs at the initial stage, followed by a polymer relaxation stage where polymer chains self-arrange for additional free volume to accommodate further sorption. Berens and Hopfenberg proposed that vapor diffusion into glassy polymers could be separated into 2 independent mechanisms, Fickian diffusion and polymer relaxation: ${ }^{4}$

$$
M_{t}=M_{\infty, F}\left[1-\sum_{n=0}^{\infty} \frac{8}{(2 n+1)^{2} \pi^{2}} \exp \left(\frac{-D(2 n+1)^{2} \pi^{2} t}{4 \ell^{2}}\right)\right]+\sum_{i} M_{\infty, i}\left[1-\exp \left(-k_{i} t\right)\right]
$$

with the first term being Fickian diffusion and the second term being polymer relaxation.

It is observed that polymer relaxation, depending on different conditions, can contribute to the mass uptake either strongly or weakly. The contributions from polymer relaxation, when mixed with diffusion and reaction contributions, emphasizes the complexity of the VPI process where all could be occurring simultaneously. Future work should involve deconvoluting the contributions from each to improve the model further. In the experimental results presented in this work, PMMA is infiltrated above its glass transition temperature, helping reduce the need for modeling of relaxation events.

The polymer is also assumed to be amorphous, homogeneous, and isotropic in composition as well as structure.

Finally, heat effects are neglected in this model, and the VPI process is assumed to be isothermal. However, the sorption of TMA into PMMA is known to be an exothermic process, thus heat should be released during the initial sorption stage. Even with the temperature controller, it would not be surprising if temperature fluctuates, especially at the initial sorption stage, which would result in a sudden change in diffusivity, reactivity, and sorption, that cannot be explained by the model as it stands. To account for heat effect, an energy balance needs to be performed. However, to properly conduct this energy balance, there must be knowledge of activation energies for diffusion, 
reaction and sorption in the VPI process of interest. As these parameters become better known in the VPI community, models encompassing these thermal effects should be developed. However, until that point in time, an isothermal VPI process is assumed to capture the main sorption kinetics and neglect the details of temperature fluctuation. 


\section{S4. Boundary Conditions for Reaction-Diffusion Model}

Initial Conditions

$C_{\text {free }}=0, @-\ell<x<\ell, t=0$

$C_{\text {product }}=0$,@- $-\ell<x<\ell, t=0$

$C_{\text {polymer }}=C_{\text {polymer }}^{0}, @-\ell<x<\ell, t=0$

Boundary Conditions

$C_{\text {free }}=C_{S}, @ x= \pm \ell, t_{\text {desorp }}>t>0$ for sorption

$C_{\text {free }}=0$,@x $= \pm \ell, t>t_{\text {desorp }}$ for desorption

$\frac{\partial C_{\text {free }}}{\partial x}=0, @ x=0, t>0$

where $C_{\text {polymer }}^{0}\left(\mathrm{~mol} / \mathrm{cm}^{3}\right)$ is the initial concentration for accessible polymeric functional groups, and $C_{S}\left(\mathrm{~mol} / \mathrm{cm}^{3}\right)$ is the surface concentration of the polymer, assumed to be at instant equilibrium with the constant vapor pressure via Henry's Law. For initial conditions, at $t=0$, within the polymer, there is no free diffusing vapor, no product formed, but accessible polymeric functional group does exist. For boundary conditions, during sorption, surface concentration will always be in equilibrium with vapor pressure according to Henry's law, and during desorption, since vapor is purged, there will be no vapor, hence no surface concentration. 


\section{S5. Non-dimensionalization of Fickian Diffusion Curves}

The well-known mass uptake response curve for Fickian diffusion is shown below, with mass uptake normalized by $M_{\infty}$, which is the equilibrium mass uptake, and time normalized by $D$ and $\ell$.

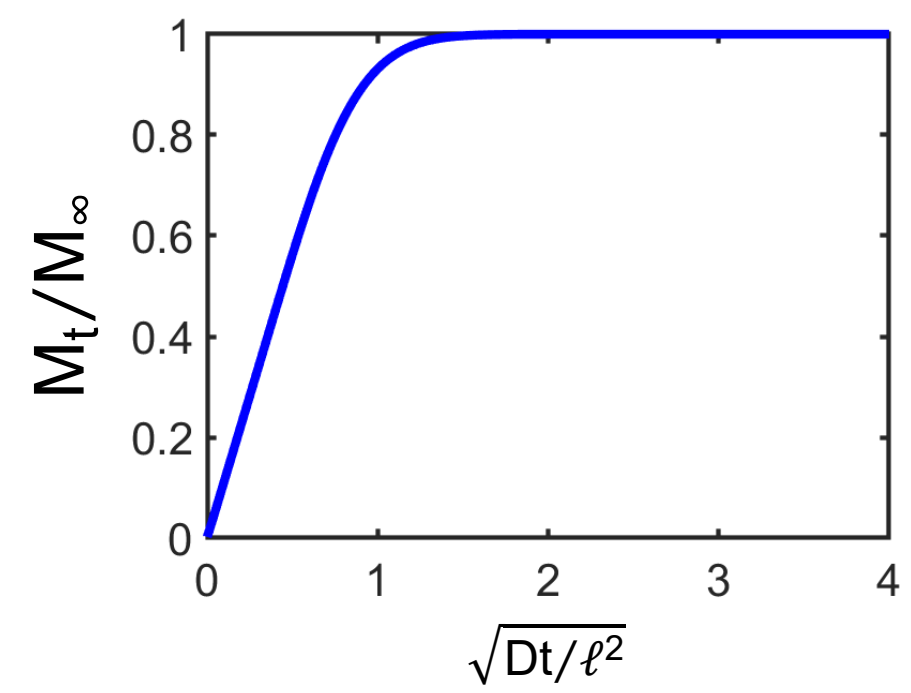

Fig S5. Fractional mass uptake vs. $\sqrt{\frac{D t}{\ell^{2}}}$ for Fickian diffusion

With the mass uptake response curve plotted by non-dimensionalized mass and time, all Fickian diffusion processes superimpose onto Figure S5. It's worth mentioning the importance of this figure, since it represents not only one specific experimental run, but all Fickian responses. The right way to check if one's vapor sorption is Fickian kinetics would be to see if the nondimensionalized experimental mass uptake curve superimposes onto this curve. The importance of non-dimensionalization starts to kick in, since the mass uptake curves, if Fickian, should be identical for different systems, regardless of what film thickness or what intrinsic diffusivity occurs in the process. The use of non-dimensionalized mass and time describes a general phenomenon, which is all Fickian diffusion, instead of just one specific operation. 


\section{S6. Non-dimensionalization of the Reaction-Diffusion Transport Model for VPI}

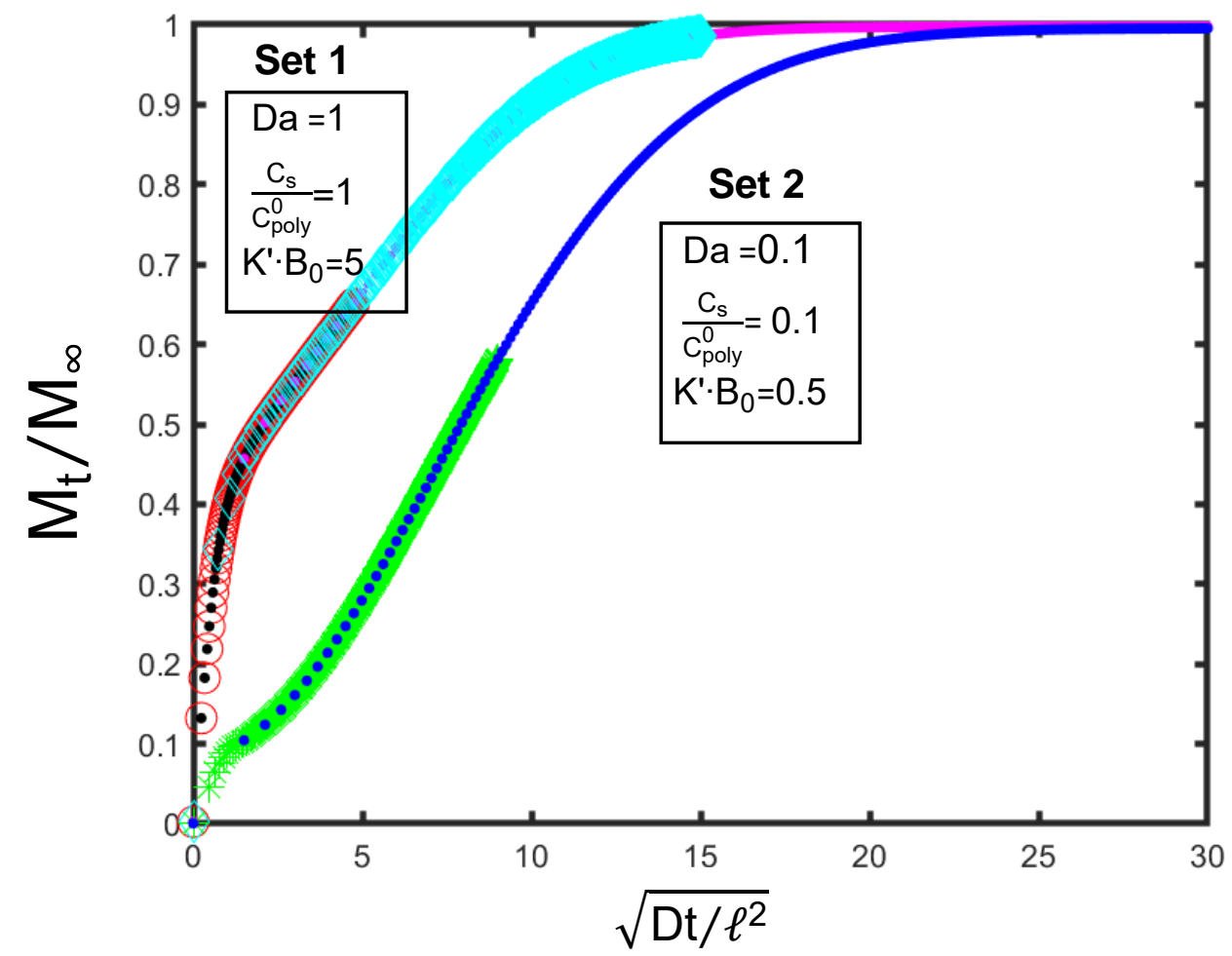

Fig S6. Non-dimensionalization curves for same dimensionless set, but different combinations of parameters. Legends of curves are summarized in Table S1.

\begin{tabular}{ccccccc}
\hline & $D_{0}$ & $k$ & $\ell$ & $C_{s}$ & $C_{\text {Poly }}^{0}$ & $K^{\prime}$ \\
\hline & & & Set 1 & & & \\
\hline$\bullet$ & 0.1 & 0.1 & 1 & 1 & 1 & 5 \\
$\bullet$ & 1 & 0.1 & 3.162 & 1 & 1 & 5 \\
& 10 & 100 & 0.3162 & 1 & 1 & 5 \\
\hline & 1 & 0.1 & 1 & 10 & 10 & 0.5 \\
\hline & 1 & 1 & 1 & 0.01 & 0.1 & 5 \\
\hline & 10 & 1 & 0.3162 & 1 & 10 & 0.05 \\
\hline
\end{tabular}

Table S1. Parameters for different legend for both Set 1 and Set 2.

From Figure S6, it can be observed that for the same dimensionless set, different combinations of parameters superimpose and give the same response. For the dimensionless Set 1 with $D a=1$, $\frac{C_{s}}{C_{P o l y}^{0}}=1$ and $K^{\prime} \cdot B_{0}=5$, even with different parameters such as varying diffusivity, reaction rate and 
polymer film thickness, they are all superimposing onto the same curve. Different curves end at different time points for clarity of the figures. 


\section{S7. Definition of $\boldsymbol{M}_{\infty}$}

$M_{\infty}$ is defined as the theoretical area normalized mass uptake that the polymer film can have assuming no impermeable layer is formed, or in other words, the total contribution of free diffusing species and immobilized product, assuming full saturation is reached. The unit of $M_{\infty}$ will be mass $/$ surface area, for instance, $\mu \mathrm{g} / \mathrm{cm}^{2}$.

Example 1: If the polymer has a thickness of $2 \mathrm{~cm}$, and the surface concentration is $2 \mu \mathrm{g} / \mathrm{cm}^{3}$ with no polymer functional groups, then as time reaches infinity, entire mass uptake will be contributed by free diffusing species, and bulk concentration will be the same as surface concentration. Thus, $M_{\infty}=2 \mu \mathrm{g} / \mathrm{cm}^{3} \times 2 \mathrm{~cm}=4 \mu \mathrm{g} / \mathrm{cm}^{2}$.

Example 2: If the polymer has a thickness of $1 \mathrm{~cm}$, and the surface concentration is $1 \mu \mathrm{g} / \mathrm{cm}^{3}$, with a polymer functional group concentration of $2 \mu \mathrm{g} / \mathrm{cm}^{3}$, then as time reaches infinity, mass uptake will be composed of free diffusing and immobilized product, thus $M_{\infty}=1 \mathrm{~cm} \times 1 \mu \mathrm{g} / \mathrm{cm}^{3}+1 \mathrm{~cm} \times$ $2 \mu \mathrm{g} / \mathrm{cm}^{3}=3 \mu \mathrm{g} / \mathrm{cm}^{2}$. 


\section{S8. Analysis of QCM Data for Experimental VPI Runs}

\begin{tabular}{|c|c|c|c|c|c|}
\hline VPI Experiment ID & $\begin{array}{c}\text { Frequency } \\
\text { of Bare } \\
\text { Crystal } \\
(\mathbf{H z})\end{array}$ & $\begin{array}{c}\text { Frequency } \\
\text { after Polymer } \\
\text { Added } \\
(\mathbf{H z})\end{array}$ & $\begin{array}{c}\text { Mass of } \\
\text { Polymer } \\
\text { Added } \\
\left(\mathbf{u g} / \mathbf{c m}^{2}\right)\end{array}$ & $\begin{array}{c}\text { Frequency } \\
\text { at End of } \\
\text { VPI Process } \\
(\mathbf{H z})\end{array}$ & $\begin{array}{c}\text { Mass Uptake } \\
\text { from VPI } \\
\left(\mathbf{u g} / \mathbf{c m}^{2}\right)\end{array}$ \\
\hline $\begin{array}{c}\text { EKM } 586 \text { TMA / PMMA } \\
130^{\circ} \mathrm{C}(8.7 \text { Torr })\end{array}$ & 5983416 & 5979020 & 54.2 & 5977366 & 20.4 \\
\hline $\begin{array}{c}\text { EKM } 612 \text { TMA / PMMA } \\
130^{\circ} \mathrm{C}(10.5 \text { Torr })\end{array}$ & 5991673 & 5986344 & 65.6 & 5984346 & 24.6 \\
\hline $\begin{array}{c}\text { EKM } 673 \text { TMA / PMMA } \\
130^{\circ} \mathrm{C}(10.4 \text { Torr })\end{array}$ & 5988099 & 5982595 & 67.8 & 5980293 & 28.4 \\
\hline $\begin{array}{c}\text { EKM } 674 \text { TMA / PMMA } \\
130^{\circ} \mathrm{C}(10.2 \text { Torr })\end{array}$ & 5986378 & 5980476 & 72.7 & 5978184 & 28.3 \\
\hline
\end{tabular}

Table S2. Frequencies measured at $130^{\circ} \mathrm{C}$ under $\sim 1.7$ Torr of flowing $\mathrm{N}_{2}$ for bare crystal (as received), after PMMA film is added via spin coating, and after VPI process where the film is now a $\mathrm{AlO}_{\mathrm{x}} / \mathrm{PMMA}$ hybrid material. Frequency values were taken as an average value of at least 15 minutes of purging under the given condition after equilibration. Mass values were calculated using the Sauerbrey relationship and are also presented for the mass of polymer added and mass uptake from the VPI process in micrograms.

\section{Outline of QCM Data Processing:}

1. Collect data from VPI experiment in a custom-built hot-walled VPI reactor containing a temperature controlled QCM.

2. Check that the change in frequency for the entire process is less than $5 \%$ (if yes, can proceed using Sauerbrey equation directly).

3. Correct mismatch from low-pressure frequency dependency. The active pumping step vs. purging step give different frequencies in the set-up described here that are artifacts of the QCM. To determine this constant value mismatch, the frequency differences between initial purging and pumping (polymer only) and final pumping and purging (hybrid material only) are averaged. This constant value is then added to all pumping regions to correct for the low pressure artifact.

4. Convert all frequencies to mass per areas using the Sauerbrey relationship:

$$
\frac{\Delta m}{A}=-\frac{\Delta f \sqrt{p_{q} \mu_{q}}}{2 f_{0}^{2}}
$$

where $\Delta m$ is the mass change in grams, $A$ is the pieozoelectrically active crystal area $\left(\mathrm{cm}^{2}\right), p_{q}$ is the density of quartz, and $\mu_{q}$ is the shear modulus of quartz.

5. Normalize all mass per areas to the total mass per area of polymer from spin-coating. 


\section{S9. Full VPI Experiments and Pressure Curves}

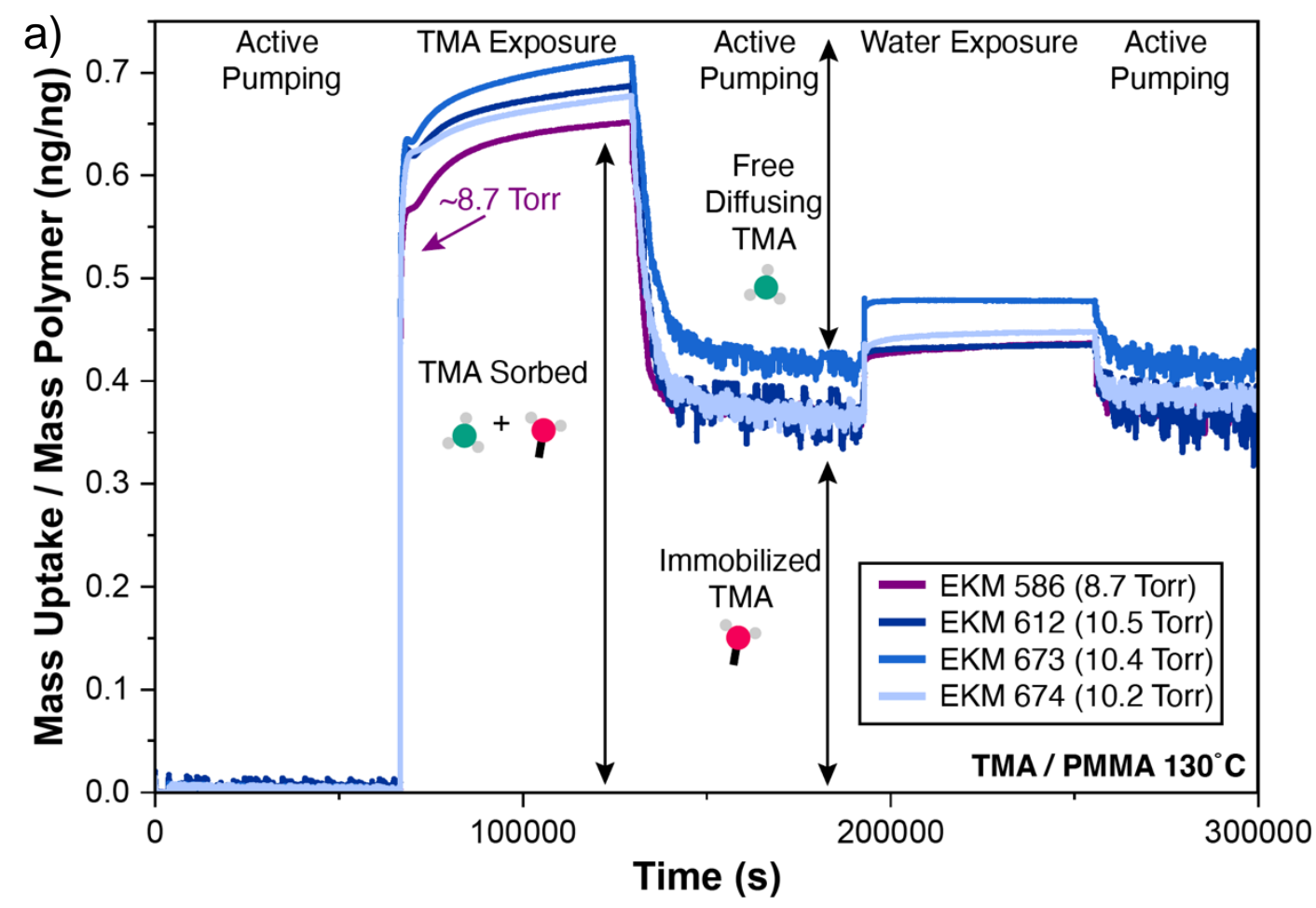

b)

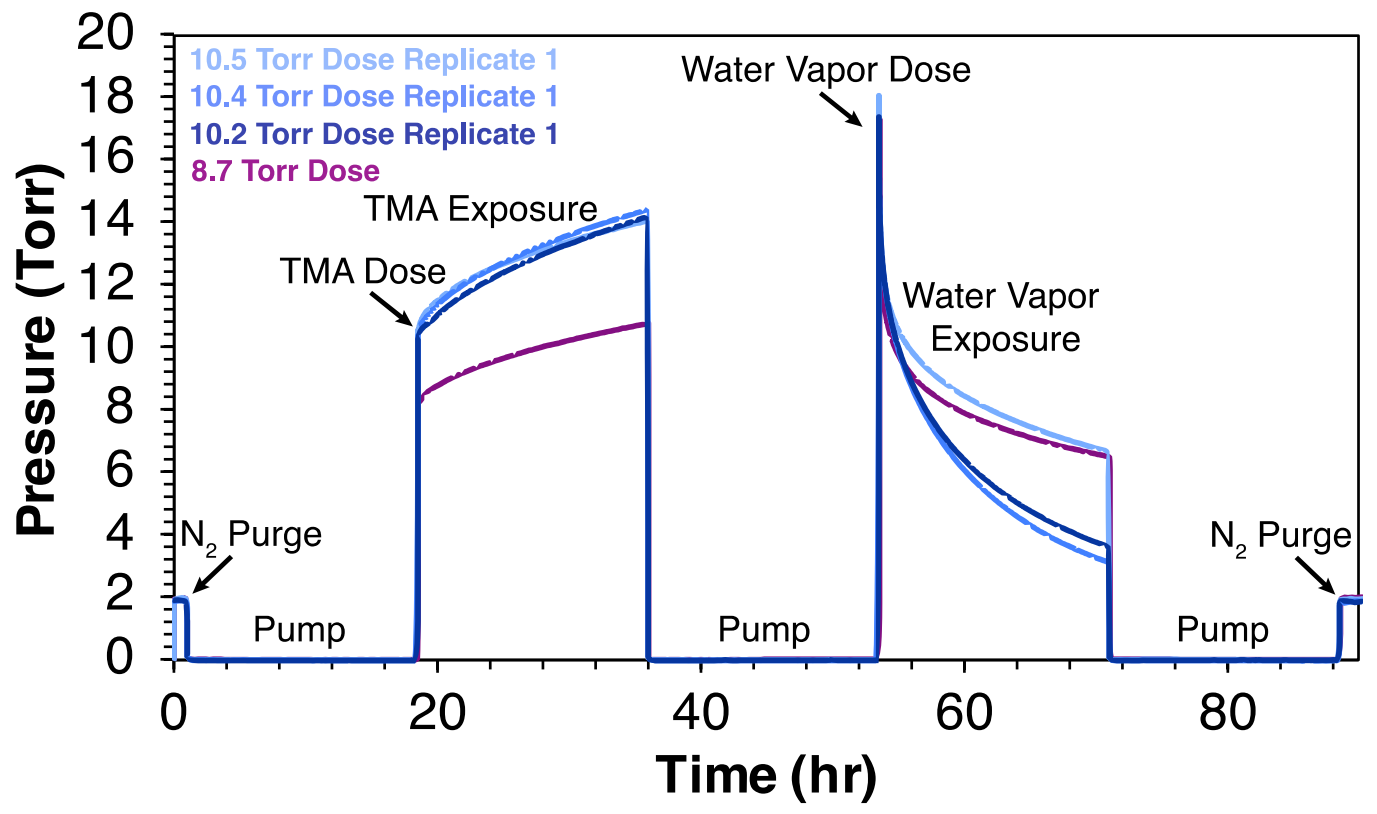

Figure S9. a) Quartz crystal microgravimetry data showing mass uptake (normalized to polymer mass) as a function of time for the infiltration of PMMA thin films with TMA at $130^{\circ} \mathrm{C}$ at $8.7,10.5,10.4$, and 10.2 Torr. b) Corresponding pressure profiles as measured by a Baratron capacitance manometer. 
A small rise of the pressure is observed during the precursor exposure step due to a chamber leak rate of $4 \mathrm{mTorr} / \mathrm{min}$. Since the partial pressure of TMA is unchanged, this small change in absolute pressure is unlikely to greatly influence the model.

\section{S10. Obtaining VPI Parameters}

\section{Thickness $\ell$}

Thickness was measured via spectroscopic ellipsometry as described in the experimental section. Diffusivity $D_{0}$

The unhindered diffusion coefficient was extracted by fitting the early time portion of the TMA uptake curve to a purely Fickian model according to the steps below:

1. Calculate mass per area vs. time according to the data processing steps 1-4 in section S8.

2. Extract data corresponding to the TMA sorption stage.

3. Normalize mass per area at each time to the total mass per area taken up in the sorption step resulting in $M_{t} / M_{\infty}$.

4. Plot $M_{t} / M_{\infty}$ for the TMA sorption stage as a function of root time.

5. Plot a Fickian curve using a summation from $n=0$ to 5 and the thickness from ellipsometry. Vary the diffusion coefficient until the modeled diffusion data fits the early time mass uptake of the experimental data reasonably well.

Surface concentration $C_{s}$

Surface concentration and accessible polymer functional group density are determined from the QCM data. The maximum mass uptake at around $250 \mathrm{~s}^{1 / 2}\left(35.149 \mu \mathrm{g} / \mathrm{cm}^{2}\right)$ is contributed by both the free diffusing species and the immobilized reaction species. When the purge is applied, the free diffusing vapor species will desorb from the polymer film, leaving the reacted immbolized metalorganic in the film $\left(19.7 \mathrm{ug} / \mathrm{cm}^{2}\right)$. Since the free diffusing mass is directly related to the surface concentration, and for free diffusing species, it is assumed that the surface concentration equals the equilibrium concentration, the surface concentration can be determined as follows:

$$
\begin{aligned}
C_{s}=\frac{\text { Mass Contribution of free diffusing species } \mu \mathrm{g} / \mathrm{cm}^{2}}{\text { Thickness of membrane }} \\
\qquad=\frac{(35.149-19.7) \mu \mathrm{g} / \mathrm{cm}^{2}}{483.1 \times 10^{-7} \mathrm{~cm}} \times \frac{10^{-6} \mathrm{~g}}{1 \mu \mathrm{g}} \times \frac{1 \mathrm{~mol}}{\frac{72.09 \mathrm{~g}}{\mathrm{~mol}}}=4.436 \times 10^{-3} \mathrm{~mol} / \mathrm{cm}^{3}
\end{aligned}
$$

Although the mass uptake is still slowly increasing at the end of the sorption stage, this change amounts to less than one percent difference $(0.2-0.3 \%)$ over the course of an hour, thus a pseudoequilibrium can be assumed and $C_{s}$ can be calculated based on the QCM data at the end of sorption. Accessible functional group density $C_{\text {polymer }}^{0}$ 
Similarly, the initial accessible polymer functional group density is also measured from the QCM, but from the mass contribution of immobilized reacted terms:

$$
\begin{aligned}
C_{\text {polymer }}^{0}=\frac{\text { Mass Contribution of reacted species } \mu \mathrm{g} / \mathrm{cm}^{2}}{\text { Thickness of membrane }} \\
=\frac{19.7 \mu \mathrm{g} / \mathrm{cm}^{2}}{483.1 \times 10^{-7} \mathrm{~cm}} \times \frac{10^{-6} \mathrm{~g}}{1 \mu \mathrm{g}} \times \frac{1 \mathrm{~mol}}{\frac{72.09 \mathrm{~g}}{\mathrm{~mol}}}=5.656 \times 10^{-3} \mathrm{~mol} / \mathrm{cm}^{3}
\end{aligned}
$$

It's important to point out that accessible functional group density, cannot be gained from stoichiometry using the polymer density and unit molecular weight. This is due to the fact that not all polymer functional groups are accessible due to steric hinderance. Even at saturation, it's possible that only half or even less of the polymer functional groups is reacted. Thus the accessible functional groups has to be gained from QCM data.

\section{Hindering factor $K^{\prime}$}

The hindering factor was determined based on the desorption curve., where it is assumed that only out-diffusion is happening. By utilizing the desorption curve, diffusion and reaction contributions can be deconvoluted. If hindering factor is 0 , desorption will be observed as extremely fast, almost like a vertical line going down. As hindering factor is gradually increased, the desorption rate will be slower due to decrease in diffusivity and the curve will be broader. Finally, there will be a value of hindering factor which result in the modeled desorption curve similar to that of the experiment. Thus, when the hindering factor $K^{\prime}$ was fitted so that the modeled desorption rate matches with the experimental data, $K^{\prime}$ was determined to be around $1150 \mathrm{~cm}^{3} / \mathrm{mol}$.

\section{Reaction rate $k$}

The reaction rate is the last parameter to be determined. With standard methods to obtain the 5 parameters from experiment data, the reaction rate constant $k$ is the only qualitative fit that is determined subjectively. When $k$ is around $1 \mathrm{~cm}^{3} / \mathrm{mol} \bullet \mathrm{s}$, the model qualitatively fits with the experimental data best. When $k=1 \mathrm{~cm}^{3} / \mathrm{mol} \bullet \mathrm{s}$, Da has a value of 0.08 . Other Da values of 0.01 , 0.1 , and 1 are also compared to show that $k=1 \mathrm{~cm}^{3} / \mathrm{mol} \bullet \mathrm{s}$ is the best fit obtained.

\section{Correction factor}

When PMMA films were spun cast onto the crystals for VPI processing, films near the edge of the crystal were removed with a cotton swab soaked in toluene to allow frontside contact to the microgravimeter, thus there will be a slight difference in the surface area of both experiments. This can be fixed easily with the correction factor. Based on calculations, the surface area of the 10.5 Torr run is $96 \%$ that of the 8.7 Torr run, thus the $96 \%$ correction factor is implemented to all mass data points in the reaction-diffusion model for 10.5 Torr prediction to capture the minor surface difference. 


\section{S.11 Parameters for Fickian diffusion and Beren's Hopfenberg}

Parameters for Beren's Hopfenberg:

The parameters for the Beren's Hopfenberg equation in S3 is as follows:

\begin{tabular}{|c|c|c|c|c|}
\hline$M_{\infty, F}\left(\mu \mathrm{g} / \mathrm{cm}^{2)}\right.$ & $D\left(\mathrm{~cm}^{2} / \mathrm{s}\right)$ & $M_{\infty, i}\left(\mu \mathrm{g} / \mathrm{cm}^{2)}\right.$ & $k_{i}(1 / \mathrm{s})$ & $l(\mathrm{~cm})$ \\
\hline 29,000 & $1 \mathrm{E}-11$ & 6000 & $7 \mathrm{E}-5$ & $483 \mathrm{E}-7$ \\
\hline
\end{tabular}

Table S3. Parameters for the Beren's Hopfenberg curve in Fig. 6.

The parameters for Fickian diffusion in S2 is as follows:

\begin{tabular}{|c|c|c|}
\hline$D\left(\mathrm{~cm}^{2} / \mathrm{s}\right)$ & $C_{S}\left(\mathrm{~mol} / \mathrm{cm}^{2}\right)$ & $l(\mathrm{~cm})$ \\
\hline $2.2 \mathrm{E}-12$ & 0.01 & $483 \mathrm{E}-7$ \\
\hline
\end{tabular}

Table S4. Parameters for the Fickian curve in Fig. 6. 


\section{S.12 Generating Dimensionless Numbers from 8.7 Torr run}

From the six parameters extracted for the 8.7 Torr run, dimensionless number sets are generated following the procedures described earlier.

The ratio of $\frac{C_{s}}{C_{\text {Polymer }}^{0}}=0.784$ indicates that surface concentration of metal-organic vapor is comparable to the concentration of the accessible polymer functional groups. The hindering degree of $K^{\prime} C_{\text {Polymer }}^{0}=6.5$ describes a moderate hindering effect from immobilized product, which we speculate is related to the polymer and vapor properties such as polymer free volume and vapor molecular size. A $D a=0.08$ indicates that there is a rapid but not instant reaction occurring between the metal-organic precursor and the polymer matrix. With a combination of comparable surface and polymer functional group concentrations, moderate hindering degree and a decent reaction rate, a non-Fickian VPI process that provides sufficient infiltration and precursor uptake can be successfully obtained. 


\section{S.13 nMAE Calculation}

Mean absolute error (MAE) is a measure of prediction accuracy for model performance evaluation. Let $\left\{\left(t_{i}^{\text {exp }}, y_{i}^{\text {exp }}\right)\right\}_{i=1}^{M}$ denotes the experimental data, and let $f$ denotes the model of interest where $f(t)$ is the model evaluation at time $t$, then the MAE is computed by

$$
\operatorname{MAE}(f)=\frac{1}{M} \sum_{i=1}^{M}\left|y_{i}^{\text {exp }}-f\left(t_{i}^{\text {exp }}\right)\right| .
$$

However, MAE alone cannot directly reflect how good the model is since MAE is scale dependent. One remedy to make this measure scale-independent is to normalize it by the average of experimental data. Let us call it the normalized MAE (nMAE),

$$
\mathrm{nMAE}=\frac{\mathrm{MAE}}{\bar{y}^{e x p}}, \bar{y}^{\text {exp }}=\frac{1}{M} \sum_{i=1}^{M} y_{i}^{e x p} .
$$

This results in a percentage, and a value closer to 0 represents a better fit. For comparison of the prediction accuracy between the sorption and desorption stage, we also compute the nMAE by normalizing each MAE with the average of all (sorption + desorption) experimental data.

Another difficulty with MAE is that the experimental data and model data are recorded at different time granularity. Since the numerical solver is expensive to compute, the model is only evaluated at 5,999 time points, in which 2,000 of them are equally spaced out between time 0 and 5,000 seconds (both end points are included) to capture the rapid increase of mass uptake at the initial stage, and the rest 3,999 time points are equally spaced out between 5,000 and 125,000 seconds (left end point is excluded), while the experimental data are recorded every half a second. Given the different time granularity, the direct computation of the nMAE is not possible. One solution is to build an interpolator $\hat{f}$ from the model data $\left\{\left(t_{i}^{\text {mod }}, y_{i}^{\text {mod }}\right)\right\}_{i=1}^{n}$, and then replace the model $f$ by the interpolator $\hat{f}$ for the nMAE computation.

Let us now discuss on how to construct an interpolator. For computational efficiency and avoiding the need of hyperparameter tuning, we decide to use the inverse distance weighting (IDW) function, that is ${ }^{5}$, for any time $t$, we have

$$
\hat{f}(t)=\left\{\begin{array}{c}
\frac{\sum_{i=1}^{n} w_{i}(t) y_{i}^{\text {mod }}}{\sum_{i=1}^{n} w_{i}(t)}, \text { if } d\left(t, t_{i}^{\text {mod }}\right)>0 \text { for all } i \\
y_{i}^{\text {mod }}, \text { if } d\left(t, t_{i}^{\text {mod }}\right)=0 \text { for some } i
\end{array},\right.
$$

where $w_{i}(t)=1 / d\left(t, t_{i}^{\text {mod }}\right)^{2}$ and $d\left(t, t_{i}^{\text {mod }}\right)$ is the Euclidean distance between time $t$ and any time $t_{i}^{\text {mod }}$ with known model evaluation $y_{i}^{\text {mod }}$. From the IDW formulation, we can see that the model estimation $\hat{f}$ on any time $t$ relies more on the nearby model evaluation points through the weight $w_{i}(t)$, and the weight decreases as the distance increases. Figure S13 shows the IDW interpolation (black solid line) performance on the actual model data (red dots). With the proposed interpolator $\hat{f}$, we can now compute the nMAE for model performance evaluation. 


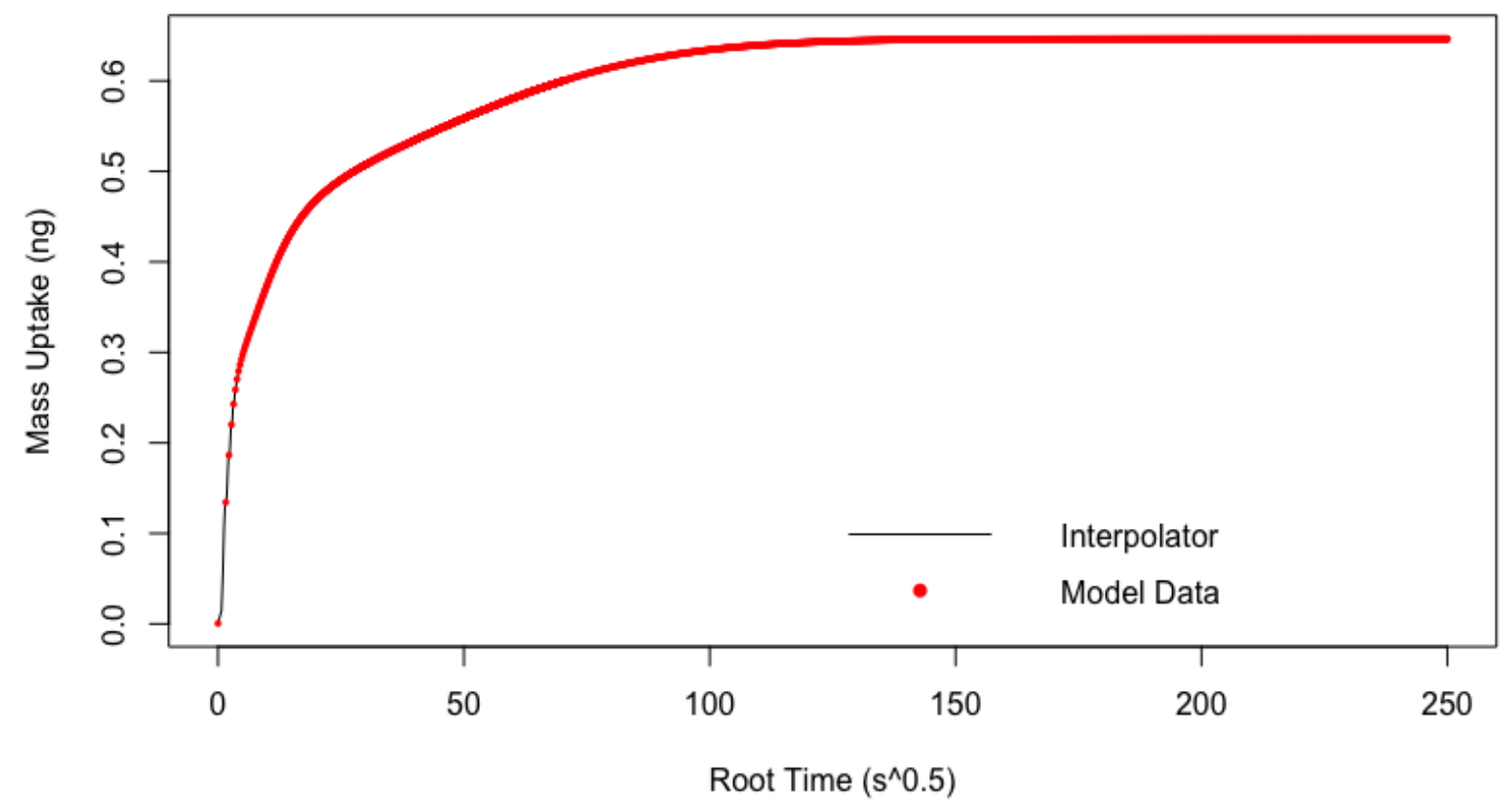

Figure S13. The inverse distance weighting (IDW) interpolation (black curve) on the actual model data (red dots). 


\section{S14. Boundary Condition for Experiment}

For the VPI experiment performed via quartz crystal microgravimetry, only one side of the polymer film is penetrated instead of two sides, as shown in Figure S14 a) and b).

a)

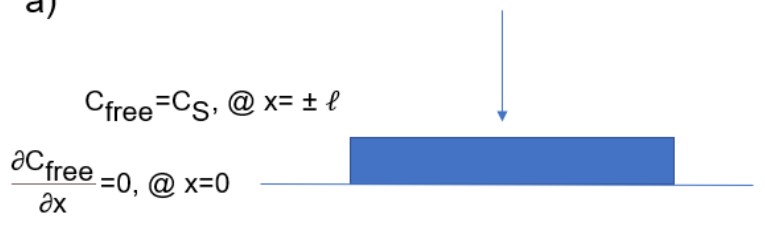

b)

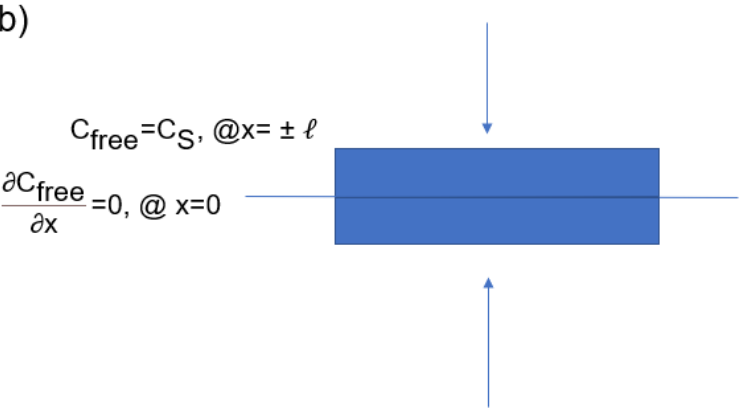

Figure S14. Comparison for two different boundary conditions for VPI experiment

The boundary condition for experimental runs is shown in Fig. S14a, where the top surface at $x=$ $\ell$ is in equilibrium with precursor vapor pressure and the bottom surface at $x=0$ is on a substrate that is impermeable such that the concentration gradient is 0 at this location. Alternatively, the boundary condition can also be simulated as shown in Fig. S14b, where the film is twice as thick with total thickness of $2 \ell$. In this case, both top and bottom surface have concentration $C_{S}$. If this boundary condition is used, then at $x=0$, there is also no concentration gradient due to a net flux of 0 at the centerline. Hence, both boundary conditions are valid for simulating the experimental VPI run since they are mathematically identical. The only thing to note is that if $b$ ) is used, the mass must be halved. Boundary condition $b$ ) is used in the paper for experimental runs since it is similar to the boundary condition used in the dimensionless mapping for Figure 3, but both boundary conditions result in identical kinetics and mass uptake response, as shown in Figure S15.

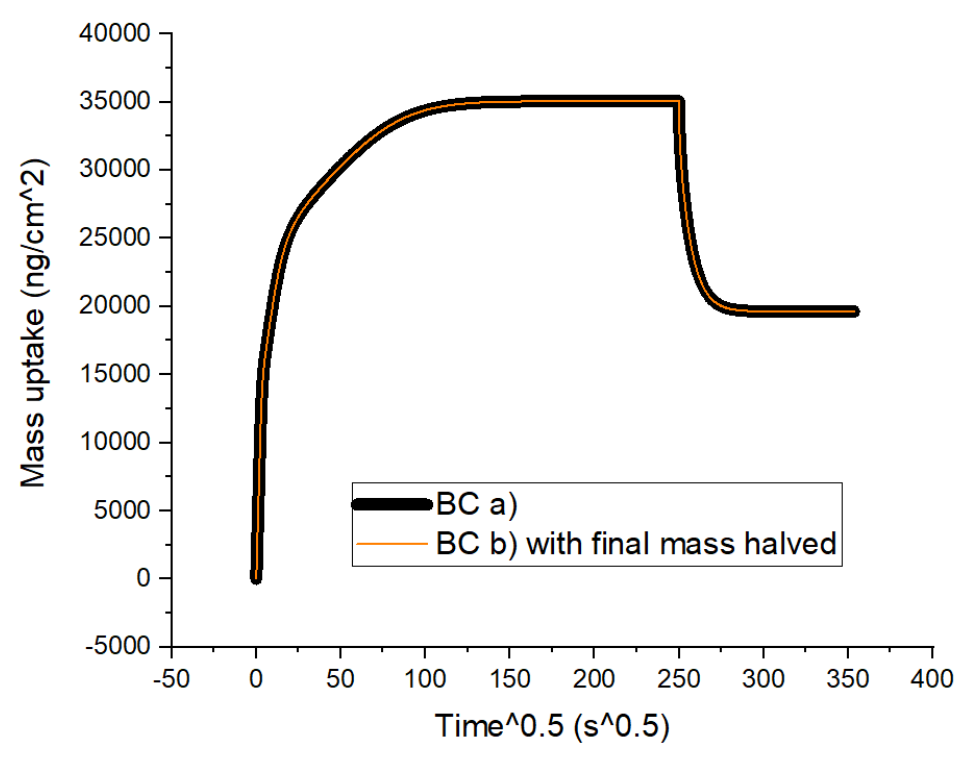


Figure S15. Mass uptake for 8.7 torr experimental run using the reaction diffusion model with 2 different boundary conditions a) and b) in Figure S14. Results are identical when the mass for b) is halved.

\section{References}

1. Leng, C. Z.; Losego, M. D., A physiochemical processing kinetics model for the vapor phase infiltration of polymers: Measuring the energetics of precursor-polymer sorption, diffusion, and reaction. Phys. Chem. Chem. Phys. 2018, 20 (33), 21506-21514.

2. $\quad$ Crank, J., The Mathematics of Diffusion. Clarendon Press: Oxford 1975.

3. Weisbord, I.; Shomrat, N.; Azoulay, R.; Kaushansky, A.; Segal-Peretz, T., Understanding and Controlling Polymer-Organometallic Precursor Interactions in Sequential Infiltration Synthesis. Chemistry of Materials 2020, 32 (11), 4499-4508.

4. Berens, A. R.; Hopfenberg, H. B., Diffusion and relaxation in glassy polymer powders 2. Separation of diffusion and relaxation parameters. Polymer 1978, 19, 489-496.

5. Shepard, D., A two-dimensional interpolation function for irregularly-spaced data. Proceedings of the 1968 ACM National Conference 1968, 517-524. 